\title{
Digital Physical Property Data for the Materials Genome Initiative
}

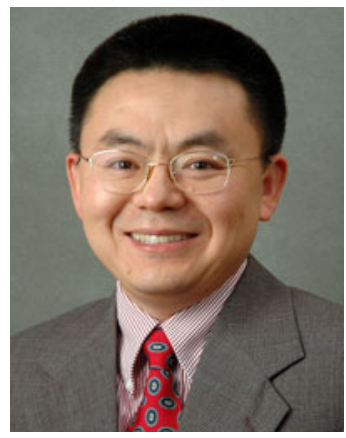

The Materials Innovation Infrastructure of the Materials Genome Initiative (MGI) announced last June by President Obama and the White House Office of Science and Technology Policy has three platforms: computational tools, experimental tools and digital data. I would like to make a few comments on digital data. As we know, thermodynamic properties and diffusivities/mobilities as a function of composition and temperature have been relatively well established using the CALPHAD approach for many materials systems and they are now widely used to predict materials compositions and processes. In contrast, composition and temperature dependent physical property data and their mathematical relations are highly deficient for even fundamental properties such as elastic modulus, thermal conductivity, thermal expansion, and optical properties. For instance, the composition-dependent thermal conductivity of the Ni-Rh system and the Ni-rich part of the Ni-Al system are shown in Fig. 1. Such data are sparse and mathematical relations to describe even the simple case of a complete solid solution such as the Ni-Rh system (including both electron and phonon contributions to the thermal conductivity) have not been widely tested for validity, not to mention the $\mathrm{Ni}$-Al system where one has to take into account the ordering effect in $\mathrm{Ni}_{3} \mathrm{Al}$ and the compositional point defects in NiAl. (The so-called Nordheim relationship is only a trend; there is no mathematical equation). Much work lies ahead to establish composition-dependent and temperature-dependent relations for thermal conductivity and several other physical properties of materials, and to build reliable digital knowledge/data-bases.

It is a much more daunting task to create knowledge/data-bases for mechanical properties. First of all, there are many kinds of mechanical properties: yield strength, ultimate strength, creep strength, fatigue properties, to name a few. Essentially all these properties are microstructure-dependent (grain size, dislocation structures, precipitate morphology and distribution, etc.) and most are test condition-dependent (strain rate, surface condition/preparation, specimen size and geometry, etc., and of course, temperature). For the data to be most valuable, all the conditions and microstructure details need to be linked to the properties. Digitization and mathematical representation of microstructure are very complicated; the D3D program funded by the Office of Naval Research has helped make significant progress not only on the front of mathematical description and reconstruction of microstructures but also experimental techniques. Even so, it is still a significant challenge to build digitized mechanical properties knowledge/data-bases. This topic is beyond the scope of this editorial.

When we try to establish databases for fundamental physical properties, we can learn a lot from the CALPHAD approach. The concept of lattice stability and the agreed-upon SGTE (Scientific Group Thermodata Europe) pure element reference stability data provide the foundation for worldwide adoption and they practically ground many databases on the same foundation. We cannot overstate the great contributions these efforts made to the advancement of the CALPHAD approach and its wide practical applications.

The framework for the diffusivity/mobility database in the CALPHAD approach is another good example for database establishment. Instead of constructing databases on diffusion coefficients by fitting polynomials through experimental
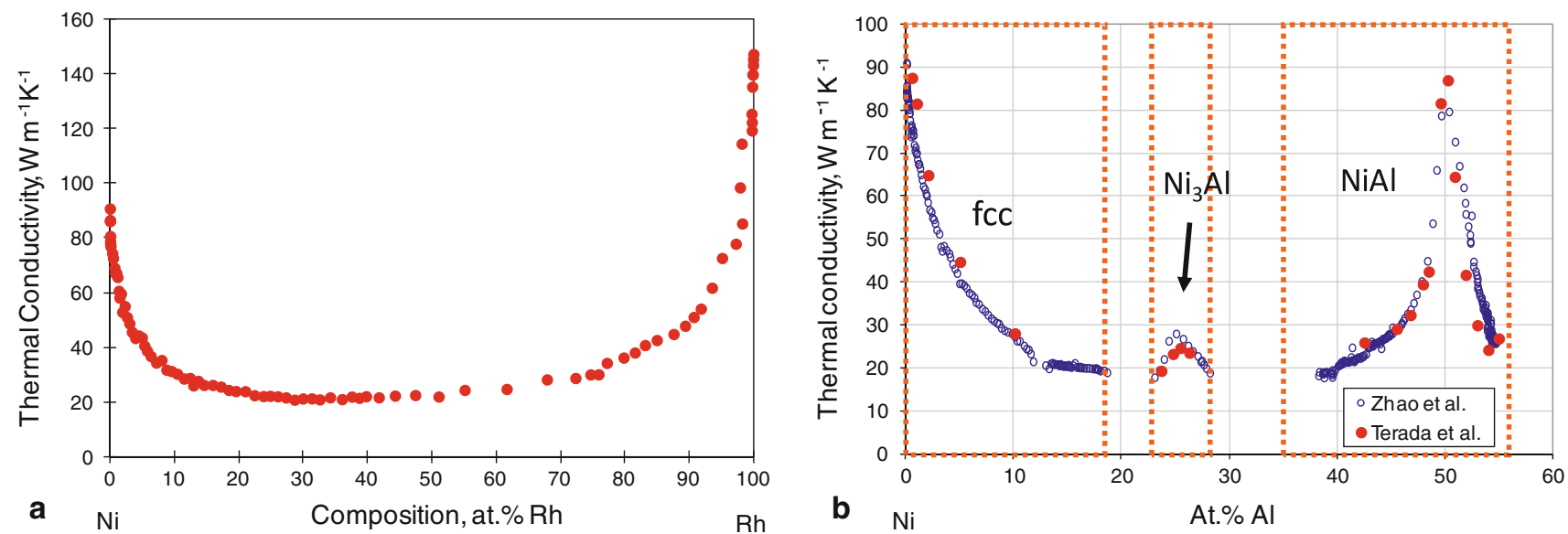

Fig. 1 Composition-dependent thermal conductivity for the Ni-Rh (a) and the Ni-Al (b) system at ambient temperature [Zhao, Prog. Mater. Sci., 51 (2006) 557; and Zhao et al., Scripta Mater., 66 (2012), 935] 
interdiffusion coefficients vs composition and temperature data, mobility databases are constructed to store the self-diffusivity/ mobility, impurity mobility, and a few parameters taking into account the non-linear behaviors. The diffusivity data can then be obtained from the Darkens' equation by taking into account the thermodynamic factor. This approach has much wider validity, especially when applied to multicomponent systems where the diffusivity matrix becomes very large and fitting polynomials for each of these diffusion coefficient data become impractical when one thinks about the fact that the each value in the diffusivity matrix is composition dependent.

When we think about reliable digital databases for physical properties, we need to first look at equations that are generally applicable to wide ranges of compositions and temperatures. Then we need to add the contributions of various effects such as magnetic transitions, ordering, and point defects among others. Since all these effects have been included in the thermodynamic assessments (or at least the CALPHAD framework allows the incorporation of these effects), it makes perfect sense to incorporate/integrate the databases for fundamental physical properties into the CALPHAD framework.

To build reliable physical property databases, we will need large amounts of experimental data not only as inputs to the digital databases, but also as validation of computational tools and methodologies. The diffusion-multiple approach and the associated micron-scale resolution property measurement tools will greatly accelerate the data gathering and reduce the need of making individual alloys for several property measurements.

For some properties such as elastic modulus, computed data from first principles are very reliable and efficient to obtain. In such a case, the experimental measurements are mostly for validation and for discovering unusual behaviors that may not have been taken into account in theoretical models. For other properties such as thermal conductivity, reliable and efficient prediction methodology has not been validated; thus the experimental measurements need to generate large amounts of data for establishing/validating the mathematical equations and also for discovering unusual behaviors so theories can be developed to account for them. In all cases, close collaborations between experimental and theoretical research will be essential for making substantial progress in establishing the framework for digital relations and databases for several physical properties.

An interesting but important question we need to ask is: whether we should bother with the CALPHAD approach at all if calculations of some of the physical properties can be done very effectively using first-principles approaches. Accurate and effective calculations of physical properties for an arbitrary composition (including multicomponent compositions) of a specific crystal structure will require algorithms that: (1) predict the physical properties to the accuracy that can be used in practical applications, (2) handle large number of atoms/unit cells for non-stoichiometric compositions or to develop effective strategy to reduce the number of unit cells while maintaining fidelity/accuracy of the computed properties, (3) take into account the various effects mentioned above (theoretically these effects should have already been "known" after first-principles calculations in a perfect world), and (4) perform accurate calculations at finite (non-0 K) temperatures by including vibrational property calculations. Tremendous progress has been made in this area and with the rapid availability of high speed computers, practical first-principles calculations are on the horizon for most physical properties. Still it will take some time before we see a multicomponent diffusion simulation that gets all the needed data by directly calling first-principles routines. In this regard, the CALPHAD approach still has its legitimacy since it is convenient, fast and accurate. A marriage of the first-principles calculations with the CALPHAD approach may be the most logical route forward.

Integration of CALPHAD predictions of compositions with predictions from property models and regression-based property databases has proven to be very effective for alloy design. A superalloy GTD262 was developed at GE in about four years from concept to production without any composition iterations using this approach; it was cited as one of the two successful examples of ICME (Integrated Computational Materials Engineering) demonstrations in a recent National Materials and Manufacturing Board report [Application of Lightweighting Technology to Military Aircraft, Vessels, and Vehichles, The National Academies Press, 2012, p. 118-119]. This example highlights the essential role of high fidelity property databases and models in accelerated development of new materials.

\author{
J.-C. Zhao \\ Associate Editor \\ Journal of Phase Equilibria anf Diffusion
}

